\title{
Die GASP als Regierungssystem: Plädoyer für einen Perspektivwechsel in der GASP-Forschung am Beispiel des EU-Kodexes zu Rüstungsexporten
}

\author{
Matthias Dembinski und Jutta Joachim*
}

Die Welle der Governance-Forschung hat das Feld der Gemeinsamen Außen- und Sicherheitspolitik (GASP) erreicht. Während einige Beiträge ,Governance‘ im Sinne von Koordinierung verstehen, ${ }^{1}$ sind andere theoretisch anspruchsvoller und konzeptualisieren den zweiten Pfeiler der Europäischen Union als ein Politikfeld, das sich durch intensive vertikale und horizontale Verflechtung, hohen Koordinationsbedarf und fehlende hierarchische Steuerungsmöglichkeiten sowie das Mitwirken einer Vielzahl von Spielern - neben Regierungsvertretern auch supranationale Organe und private Akteure - auszeichnet. ${ }^{2}$

Mit dieser Wendung reagiert die Forschung zum einen auf beobachtbare Veränderungen institutioneller Strukturen: nämlich die wachsende Präsenz und die zunehmenden Mitsprachewünsche und -möglichkeiten von Nichtregierungsorganisationen (NGOs) und Interessenvertretern im Bereich der Sicherheits- und Verteidigungspolitik auf europäischer Ebene einerseits sowie die mit der Europäischen Sicherheits- und Verteidigungspolitik (ESVP) stark beschleunigte ,Brüsselisierung ' und Institutionalisierung des zweiten Pfeilers andererseits. $^{3}$

Zum anderen vollzieht die Forschung zur GASP damit eine akademische Trendwende nach, die im Bereich der Binnenmarktforschung bereits in den 1990er Jahren zur Zurückdrängung staatszentrierter Ansätze zugunsten von Modellen wie dem des Mehrebenensystems ${ }^{4}$ und des Regierens in Netzwerken ${ }^{5}$ führte. Unter dem Schlagwort ,Governance“ hat sich seitdem eine gesellschaftsorientierte theoretische Perspektive etabliert, deren Vertreter/ -innen mehrheitlich davon ausgehen, dass europäische Entscheidungen nicht mehr exklusiv von Regierungen getroffen, sondern in einem Neben- und Miteinander von Regierungen, supranationalen Organen, nationalen Legislativen und regionalen Gebietskörperschaften sowie von privaten Interessengruppen und NGOs ausgehandelt und umgesetzt werden.

* Dr. Matthias Dembinski, Hessische Stiftung Friedens- und Konfliktforschung, Frankfurt am Main. PD Dr. (Ph. D.) Jutta Joachim, Leibniz Universität Hannover.

1 Siehe beispielsweise Sandra Lavenex: EU External Governance in ,Wider Europe', in: Journal of European Public Policy 4/2004, S. 680-700 oder Simon Duke/Sophie Vanhoonacker: Administrative Governance in the CFSP: Development and Practice, in: European Foreign Affairs Review 2/2006, S. 163-182.

2 Mark Webber/Stuart Croft/Jolyon Howorth/Terry Terriff/Elke Krahmann: The Governance of European Security, in: Review of International Studies 1/2004, S. 3-26; Emil J. Kirchner: The Challenge of European Union Security Governance, in: Journal of Common Market Studies 5/2006, S. 947-968; Sebastian Mayer: Prävention und Intervention: Security Governance im Mehrebenensystem der Europäischen Union, in: Zeitschrift für Politikwissenschaft 1/2008, S. 79-103.

3 David Allen: 'Who Speaks for Europe?'. The Search for an Effective and Coherent External Policy, in: John Peterson/Helen Sjursen (Hrsg.): A Common Foreign Policy for Europe? Competing visions of the CFSP, London 1998, S. 41-58.

4 Gary Marks/Liesbet Hooghe/Kermit Blank: European Integration from the 1980s: State-Centric v. Multi-Level Governance, in: Journal of Common Market Studies 3/1996, S. 341-378.

5 Dorothea Jansen/Klaus Schubert (Hrsg.): Netzwerke und Politikproduktion. Konzepte, Methoden, Perspektiven, Marburg 1995; Tanja A. Börzel: What's So Special About Policy-Networks? An Exploration of the Concept and Its Usefulness in Studying European Governance, European Integration Online Papers 16/1997; Beate Kohler-Koch/Rainer Eising (Hrsg.): Transformation of Governance in the European Union, London 1999. 
Die Öffnung der GASP-Forschung für gesellschaftsorientierte Ansätze überwindet damit ein Schisma, das die Integrationsforschung bis vor wenigen Jahren prägte. Denn in der zeitgeschichtlichen ${ }^{6}$ und politikwissenschaftlichen ${ }^{7}$ Literatur zur europäischen Außenpolitik dominierten staatszentrierte Ansätze. Im Falle von Beiträgen, die sich eines realistischen Ansatzes $^{8}$ bedienten, mag dies nicht überraschen. Aber selbst Studien, die die GASP mithilfe eines neo-funktionalistischen ${ }^{9}$ oder konstruktivistischen Ansatzes ${ }^{10}$ analysierten, verkürzten den Blickwinkel im Wesentlichen auf die Sozialisation von Regierungsvertretern im Kommunikationssystem der GASP. Umgekehrt konzentrierten sich die Vertreter/-innen der Zunft, die sich einer gesellschaftsorientierten Perspektive verpflichtet sahen, auf die Politikfelder des ersten Pfeilers und ignorierten den Bereich des zweiten Pfeilers weitgehend. Symptomatisch für diese Blindstelle ist die vollständige Ausblendung der Außen- und Sicherheitspolitik in dem Standardwerk zur Interessenrepräsentation in der Europäischen Union. ${ }^{11}$

Wir halten die Öffnung der GASP-Forschung für gewinnversprechend. Denn sowohl die zunehmende Institutionalisierung als auch das zivilgesellschaftliche Engagement in der zweiten Säule lassen sich kaum mehr mit einer ausschließlich staatszentrierten Sichtweise erklären und werfen zumindest die Frage nach einem Perspektivenwechsel auf. Dennoch warnen wir vor einer schnellen und unreflektierten Übernahme des Governance-Ansatzes. Zum einen sind die im Zusammenhang mit diesem Ansatz verwandten Definitionen unscharf, weshalb Kritiker monieren, dass hier unter dem Mantel neuer Begrifflichkeiten alte Theoreme verkauft würden. ${ }^{12}$ Zum anderen postuliert diese Forschung oftmals nur, was erst nachzuweisen wäre: nämlich dass die Beteiligung von Institutionen und privaten Akteuren bedeutsam ist und die Ergebnisse des politischen Prozesses signifikant beeinflusst. Schließlich wäre zu prüfen, ob das Governance-Pferd nur auf dem Parcour des ersten Pfeilers gut läuft beziehungsweise inwieweit mögliche strukturelle Unterschiede zwischen dem ersten und zweiten Pfeiler die Erklärungskraft dieses Modells im Bereich der Außen- und Sicherheitspolitik trüben.

Um die Fruchtbarkeit von Governance-Ansätzen in der GASP auszuloten, gehen wir in drei Schritten vor: Wir beginnen mit einer Rekonstruktion der Unterschiede zwischen staatszentrierten Ansätzen und einer Governance-Perspektive und entwickeln darauf aufbauend eine Forschungsstrategie, die es erlaubt, den Effekt der Beteiligung privater Akteure und supranationaler Institutionen zu erfassen. In einem zweiten Schritt diskutieren wir dann kurz die Voraussetzungen der Anwendbarkeit des Governance-Begriffs in der GASP. Daran anschließend leuchten wir am Beispiel der Entwicklung des europäischen Verhaltenskodexes zur Kontrolle von Rüstungsexporten die Bedeutung von Institutionen und das Engagement von NGOs unter besonderer Berücksichtigung ihrer Strategien und ihrer Wirkung auf den politischen Prozess aus. Auf dieser Grundlage diskutieren wir abschließend, welche Muster

6 Simon Nuttall: European Foreign Policy, Oxford 2000.

7 Elfriede Regelsberger: Die Gemeinsame Außen- und Sicherheitspolitik der EU (GASP). Konstitutionelle Angebote im Praxistext 1993-2003, Baden-Baden 2004.

8 Stanley Hoffmann: Obstinate or Obsolete? The Fate of the Nation State and the Case of Western Europe, in: Daedalus 3/1966, S. 862-915; Werner Link: Die Entwicklungstendenzen der Europäischen Integration (EG/ EU) und die neo-realistische Theorie, in: Zeitschrift für Politik 3/2001, S. 302-321; Barry R. Posen: ESDP and the Structure of World Power, in: The International Spectator 1/2004, S. 5-17.

9 Jakob C. Øhrgaard: 'Less than Supranational, More than Intergovernmental': European Political Cooperation and the Dynamics of Intergovernmental Integration, in: Millennium - Journal of International Studies 1/1997, S. 1-29.

10 Michael E. Smith: Europe's Foreign and Security Policy: The Institutionalization of Cooperation, Cambridge 2003.

11 Justin Greenwood: Interest Representation in the European Union, Basingstoke 2003.

12 Siehe beispielsweise Klaus Dingwerth/Philipp Pattberg: Was ist Global Governance?, in: Leviathan 3/2006, S. 377-399. 
sich erkennen lassen, welche Gemeinsamkeiten und Unterschiede zwischen dem ersten und dem zweiten Pfeiler bestehen und welche Folgen sich daraus für das Verständnis der GASP sowie die zukünftige Forschung ableiten lassen.

\section{Staatszentrierte versus Governance-Ansätze in der EU-Forschung}

Staatszentrierte Ansätze sehen in den Staaten oder genauer den Spitzen ihrer Exekutiven die treibende Kraft der Integration. Deren Interessen und Verhandlungsmacht bestimme den Gang und die Ergebnisse der politischen Entscheidungsprozesse auf europäischer Ebene. Supranationale Akteure wie die Europäische Kommission werden als Agenten der Staaten konzeptualisiert, die zwar eigene Interessen verfolgen können, aber letztlich der Kontrolle ihrer Prinzipale unterliegen. ${ }^{13}$ Gesellschaftliche Akteure spielen nur insofern eine Rolle, als sie zum einen auf der Ebene der nationalen Entscheidungsprozesse an der Ausarbeitung staatlicher Positionen mitwirken, auf der anderen Seite zum Adressat der auf europäischer Ebene getroffenen Beschlüsse werden. ${ }^{14}$ Derartige Zwei-Ebenen-Modelle europäischer Politik weisen den Vertretern der Exekutiven als Schnittstellenakteure besondere Macht zu, weil sie den Transfer von Information zwischen der supranationalen und der nationalen Ebene regulieren und gemäß ihrer eigenen Interessen auf beiden Ebenen manipulieren können. ${ }^{15}$

Governance-Modelle zeichnen sich dagegen durch die Berücksichtigung weiterer Akteure und anderer Formen kollektiven Handelns aus. Allerdings sieht sich jeder Versuch einer Würdigung der Erklärungskraft dieser Modelle in Konkurrenz zu staatszentrierten Ansätzen mit dem Problem einer unzureichenden Präzision und Trennschärfe des Begriffs ,Governance“ konfrontiert. Eine weite Fassung sieht ,Governance“ als „Oberbegriff für sämtliche vorkommenden Muster der Interdependenzbewältigung“. ${ }^{16}$ Dagegen will ein engeres Verständnis den Governance-Begriff für Formen zielgerichteter und auf Dauer angelegter Kooperation reservieren, die sich durch zwei weitere Merkmale auszeichnen: zum einen Komponenten der Normorientierung und diskursiven Verständigung, zum anderen die Einbeziehung nichtstaatlicher Akteure. In diesen Definitionen spiegelt sich die Verwurzelung des Governance-Begriffs in den Internationalen Beziehungen einerseits, sowie der Policy-Forschung andererseits wider.

Die Ursprünge des weiten Governance-Begriffs lassen sich in den Internationalen Beziehungen bis zur Debatte um die Möglichkeiten institutionalisierter Koordination von Staaten unter der Bedingung der Anarchie in den 1980er Jahren zurückverfolgen. ${ }^{17}$ Der enge Begriff hingegen setzt auf die Steuerungsdebatten auf, die in der Policy-Forschung ab den 1970er Jahren geführt wurden. ${ }^{18}$ Auf die offenkundiger werdenden Grenzen der hierarchischen

13 Mark A. Pollack: Delegation, Agency and Agenda Setting in the European Union Community, in: International Organization 1/1997, S. 99-134.

14 Andrew Moravcsik: Preferences and Power in the European Community: A Liberal Intergovernmentalist Approach, in: Journal of Common Market Studies 4/1993, S. 473-524.

15 Robert D. Putnam: Diplomacy and Domestic Politics: the Logic of Two-Level Games, in: International Organization 3/1988, S. 427-460; Bernhard Zangl: Der Ansatz der Zwei-Ebenen-Spiele. Eine Brücke zwischen dem Neoinstitutionalismus und seinen KritikerInnen?, in: Zeitschrift für Internationale Beziehungen 2/1995, S. 393-416.

16 Arthur Benz/Susanne Lütz/Uwe Schimank/Georg Simonis: Einleitung, in: Arthur Benz/Susanne Lütz/Uwe Schimank/Georg Simonis (Hrsg.): Handbuch Governance. Theoretische Grundlagen und empirische Anwendungsfelder, Wiesbaden 2007, S. 9-25.

17 Markus Jachtenfuchs: Regieren jenseits der Staatlichkeit, in: Gunther Hellmann/Klaus Dieter Wolf/Michael Zürn (Hrsg.): Die neuen Internationalen Beziehungen. Forschungstand und Perspektiven in Deutschland, Baden-Baden 2003, S. 495-518, hier S. 497.

18 Renate Mayntz: Politische Steuerung: Aufstieg, Niedergang und Transformation einer Theorie, in: Klaus von Beyme/Claus Offe (Hrsg.): Politische Theorien in der Ära der Transformation, Politische Vierteljahresschrift Sonderheft 26/1995, S. 148-168; Josef Esser: Der kooperative Nationalstaat im Zeitalter der „Globalisierung“, in: Diether Döring (Hrsg.): Sozialstaat in der Globalisierung, Frankfurt am Main 1999, S. 117-144. 
Steuerung gesellschaftlicher Teilbereiche durch zentrale Regierungen reagierte die Forschung mit der Thematisierung von Modellen der zielgerichteten Koordinierung öffentlicher und privater Akteure. ${ }^{19} \mathrm{Um}$ unter den Bedingungen zunehmender Komplexität und Wissensintensität, der strukturellen Informationsdefizite zentralstaatlicher Steuerungsinstanzen sowie der Autonomie und Resistenz privater Akteure und gesellschaftlicher Teilsysteme überhaupt die Hoffnung auf politische Steuerung und allgemeinverbindliche Entscheidungen gegen die neo-liberale Forderung nach mehr Markt aufrecht erhalten zu können, setzte die politikwissenschaftliche Forschung auf Modelle des verhandelnden Staates, der in Kooperation mit semi-autonomen gesellschaftlichen Akteuren gemeinwohlverträgliche Lösungen sucht. Die im Rahmen dieser Modelle problemlos denkbare Entterritorialisierung des Regierens machte dieses Verständnis von Goverance zum idealen Kandidaten, um beobachtbare Muster der Koordinierung auf den Feldern des europäischen Binnenmarktes theoretisch abzubilden.

Im Rahmen dieser Studie definieren wir ,Governance“ dem engen Verständnis folgend als ebenenübergreifenden Koordinierungs- und Verhandlungsprozess zwischen öffentlichen und privaten Akteuren zur Produktion allgemeinverbindlicher Entscheidungen. Damit unterscheiden wir uns von den bisherigen Versuchen, Governance-Modelle in der GASP-Forschung fruchtbar zu machen. So sind beispielsweise sowohl Webber et. al. ${ }^{20}$ als auch Kirchner ${ }^{21}$ dem weiten Governance-Begriff verpflichtet. Sie reduzieren Governance auf die institutionalisierte Kooperation von Staaten, die sie weiterhin als dominante Akteure der GASP begreifen. Dementsprechend spielen private Akteure in beiden Untersuchungen eine untergeordnete Rolle. Der Unterschied zu staatszentrierten Modellen beschränkt sich damit auf die These, die staatliche Kooperation sei von Normen und Ideen gesteuert. Mit der Verwendung eines engen Verständnisses des Governance-Begriffs lassen sich dagegen die Unterschiede in den Erwartungen staatszentrierter und Governance-Ansätze deutlicher herausarbeiten.

Ein erster Unterschied betrifft Kernfragen des Forschungsdesigns. Staatszentrierte Ansätze konzeptualisieren Interessen und Verhandlungsmacht der Staaten als unabhängige, europäische Entscheidungen über verteilungspolitische oder institutionelle Fragen als abhängige Variable. Dagegen wird in Governance-Ansätzen die europäische Polity als zu erklärendes Phänomen konzeptualisiert und die Europäisierung nationaler Politik als eine Begründung dafür. ${ }^{22}$

Ein zweiter Unterschied betrifft die Akteure und die Konsequenzen der Europäisierung für ihre Stellung untereinander. Staatszentrierte Modelle sehen die Staats- und Regierungschefs der Mitgliedsländer als ausschlaggebende Akteure. Sie erwarten im Prozess der Integration eine Umverteilung politischer Macht von Gesellschaften und Legislativen hin zu Exekutiven und damit zusammenhängend eine Einschränkung der Transparenz politischer Prozesse und Entscheidungen. ${ }^{23} \mathrm{Im}$ Gegensatz dazu basieren Governance-Modelle auf der Annahme, dass sowohl gesellschaftliche Akteure als auch regionale und supranationale politische Akteure mit autonomen Ressourcen ausgestattet sind und die europäischen Entschei-

19 Renate Mayntz: Governance im modernen Staat, in: Arthur Benz (Hrsg.): Governance - Regieren in komplexen Regelsystemen, Wiesbaden 2004, S. 65-76.

20 Webber/Croft/Howorth/Terriff/Krahmann: The Governance of European Security, 2004.

21 Kirchner: The Challenge of European Union Security Governance, 2006.

22 Markus Jachtenfuchs: The Governance Approach to European Integration, in: Journal of Common Market Studies 2/2001, S. 245-264, hier S. 245.

23 Andrew Moravcsik: Why the European Union Strengthens the State: Domestic Politics and International Institutions, in: Center for European Studies Working Paper Series 52/1994; Klaus Dieter Wolf: Die neue Staatsräson: Zwischenstaatliche Kooperation als Demokratieproblem in der Weltgesellschaft, Baden-Baden 2000. 
dungsprozesse beeinflussen. ${ }^{24}$ Anders als staatszentrierte Ansätze hegen sie Zweifel an der Behauptung, dass sich die europäische und die nationale Ebene sauber voneinander trennen ließen. Marks, Hooghe und Blank räumen zwar ein, die Staaten seien ,an integral and powerful part of the EU“, weisen aber einschränkend darauf hin, ,they no longer provide the sole interface between supranational and sub-national arenas, and they share, rather than monopolize, control over many activities that take place in their respective territories". 25 Nicht nur Exekutiven, auch private Akteure, Parlamentarier und Vertreter regionaler Gebietskörperschaften sind auf der europäischen Ebene präsent, begleiten und überwachen europäische Prozesse und formen über die nationalen Exekutiven hinweg Koalitionen mit supranationalen Akteuren und/oder Vertretern anderer Staaten. Ein Verlust an Einflussmöglichkeiten seitens nationaler Parlamente sowie zivilgesellschaftlicher Akteure ist daher nicht zu erwarten.

Ein dritter Unterschied betrifft die Formen und Ergebnisse der Koordinierung. Aus der Sicht staatszentrierter Modelle wird das Ergebnis der Verhandlungen wesentlich von dem Staat bestimmt, der seine Ziele am ehesten alleine oder außerhalb der Europäischen Union realisieren kann beziehungsweise am wenigsten Anreize für eine Änderung des Status quo hat. Nach Auffassung von Governance-Modellen hingegen wird die Verhandlungsmacht der Staaten mehrfach gebrochen. Zum einen können institutionelle Rollen den Einfluss einzelner Staaten begrenzen oder vergrößern. Zum anderen beeinflusst die Beteiligung von NGOs und supranationalen Organen die Ergebnisse des Entscheidungsprozesses. Dabei sieht die Governance-Forschung die Europäische Kommission, das Europäische Parlament oder den Europäischen Gerichtshof nicht nur als Agenten der Staaten, sondern im Einklang mit der neueren institutionalistischen Forschung als Bürokratien, ${ }^{26}$ die ihre eigene Handlungslogik ausprägen und als Akteure den politischen Prozess beeinflussen. ${ }^{27}$ Darüber ändert sich mit der Institutionalisierung und der Beteiligung nichtstaatlicher Akteure der Charakter der Entscheidungsprozesse. Weil die Verhandlungen von Dauer sind und auf einer öffentlichen Bühne stattfinden, spielt die Abwägung der Gemeinwohlverträglichkeit von vorgeschlagenen Lösungen im Lichte der verfügbaren Informationen, die Schlüssigkeit der Argumente und die Vereinbarkeit der Verhandlungspositionen mit gemeinsam geteilten Normen, Prinzipien und Regeln eine große Rolle.

Auf der Grundlage dieser theoretischen Erwartungen lässt sich eine Forschungsstrategie entwickeln, um die Bedeutung der Institutionalisierung und der Beteiligung privater Akteure für die Ergebnisse des politischen Prozesses zu erfassen. Dabei werden zunächst die oben genannten Erwartungen aus staatszentrierter Perspektive entsprechend der Bedingungen des zu untersuchenden Feldes genauer spezifiziert. Wenn Differenzen zwischen den theoretischen Erwartungen und empirischen Beobachtungen festgestellt werden, lässt sich zweitens anhand einer Rekonstruktion politischer Prozesse prüfen, inwieweit die Institutionalisierung und die Mitwirkung privater Akteure diese Differenz erklären. Bevor wir den heuristischen Wert dieser Strategie am Beispiel der Entstehung des europäischen Verhaltenskodexes zur Kontrolle von Rüstungsexporten illustrieren, sollen zunächst die formalen Voraussetzungen der Anwendbarkeit von Governance-Modellen in der GASP geprüft werden.

24 Edgar Grande: Multi-Level Governance: Institutionelle Besonderheiten und Funktionsbedingungen des europäischen Mehrebenensystems, in: Edgar Grande/Markus Jachtenfuchs (Hrsg.): Wie problemlösungsfähig ist die EU? Regieren im europäischen Mehrebenensystem, Baden-Baden 2000, S. 11-30.

25 Marks/Hooghe/Blank: European Integration from the 1980s, 1996, S. 347.

26 Michael N. Barnett/Martha Finnemore: Rules for the World. International Organizations in Global Politics, Ithaca 2004.

27 Duke/Vanhoonacker: Administrative Governance in the CFSP, 2006. 


\section{Nichtstaatliche Akteure und die GASP: empirische Beobachtungen}

Die Außenpolitik gilt traditionell als Domäne der Regierung, die im Interesse der Staatsraison dem Einfluss der Interessengruppen und supranationaler Organe entzogen ist und zu entziehen sei. Auch wenn dieses Konzept einem Wandel unterliegt und die klassische Diplomatie längst einem Verständnis von Außenpolitik Platz gemacht hat, dem es sowohl um die Beeinflussung von Gesellschaften in anderen Ländern geht als auch um das Mitspracherecht der eigenen Gesellschaft, ${ }^{28}$ wäre dennoch zu fragen, inwieweit die institutionellen Bedingungen im zweiten Pfeiler besondere sind und den Zugang von Interessengruppen sowie den Einfluss von Institutionen beschränken. Es soll geprüft werden, ob sich (1) NGOs und Interessengruppen auf europäischer Ebene engagieren, (2) welche Rolle dabei die Institutionalisierung und die Organe der Europäischen Union spielen und (3) inwiefern sich das Engagement zivilgesellschaftlicher Akteure im zweiten Pfeiler von dem im ersten unterscheidet.

Der erste Nachweis ist einfach zu führen. Tatsächlich verlagerten in Übereinstimmung mit der von Kohler-Koch ${ }^{29}$ und anderen ${ }^{30}$ vertretenen Ko-Evolutionsthese außen- und sicherheitspolitisch interessierte NGOs und Interessengruppen in dem Maße ihre Aktivitäten von der nationalen auf die europäische Ebene, in dem die Europäische Union auf diesen Feldern politische Steuerungskompetenz gewann.

Besonders ausgeprägt ist das Engagement der NGOs im Bereich der Entwicklungshilfe und des Katastrophenschutzes, der Menschenrechtspolitik und der internationalen Umweltpolitik. Aber selbst im Kernbereich der Sicherheitspolitik ist die GASP längst keine verbands- und öffentlichkeitsfreie Zone mehr und begleiten eine Reihe von NGOs und anderen Interessengruppen europäische Außenpolitik.

Lokale friedenspolitische Graswurzel-Organisationen und NGOs bildeten in den 1990er Jahren europäische Netzwerke wie das European Network for Civil Peace Services (EN.CPS), die European Platform for Conflict Prevention and Transformation oder den europäischen Arm des International Action Network on Small Arms (IANSA). Diese Zusammenschlüsse verfolgten die Ziele, die Kontakte untereinander zu stärken und gemeinsam europäische Politik zu beeinflussen und umgekehrt die Gruppen auf lokaler Ebene über friedens- und abrüstungspolitisch relevante Prozesse und Entscheidungen auf europäischer Ebene zu informieren. Neben Netzwerken europäischer Graswurzel-Organisationen betreiben eine Reihe professioneller, transnationaler Organisationen in Brüssel friedens- und abrüstungspolitische Lobbyarbeit. Hierzu zählen Einrichtungen mit kirchlichem Hintergrund wie das Quaker Council for European Affairs, Pax Christi International oder World Vision sowie Organisationen mit weltlicher Orientierung, die wie Greenpeace oder Oxfam im Schnittfeld von friedens-, umwelt- und entwicklungspolitischer Arbeit aktiv sind. Schließlich haben sich Akteure im Grenzbereich von NGOs und Think Tanks in Brüssel angesiedelt. Besonderer Resonanz erfreuen sich die International Crisis Group oder ISIS Europe, ein 1995 vom Londoner International Security Information Service gegründeter Ableger. Besondere Erwähnung verdient das European Peacebuilding Liason Office (EPLO), ein 2001 gegründeter Zusammenschluss von heute 22 NGOs, Think Tanks und Netzwerken.

28 Christopher Hill: The Changing Politics of Foreign Policy, Basingstoke 2003.

29 Beate Kohler-Koch: Die Gestaltungsmacht organisierter Interessen, in: Markus Jachtenfuchs/Beate KohlerKoch (Hrsg.): Europäische Integration, Opladen 1996, S. 193-222.

30 Volker Eichener/Helmut Voelzkow: Europäische Integration und verbandliche Interessenvermittlung, Marburg 1994; Alec Stone Sweet/Wayne Sandholtz: European Integration and Supranational Governance, in: Journal of European Public Policy 3/1997, S. 297-317. 
Neben der Präsenz friedenspolitisch ausgerichteter Organisationen ist ein geradezu boomartiges Wachstum von außen- und sicherheitspolitischen Expertennetzwerken festzustellen. Hierzu zählen die schon seit Längerem in Brüssel ansässigen und ursprünglich auf binnenmarktpolitische Themen spezialisierten Einrichtungen wie das Centre for European Policy Studies (CEPS) und das European Policy Centre (EPC). Daneben entstanden eine Reihe neuer Einrichtungen. Die 2002 ins Leben gerufene Security \& Defence Agenda (SDA) befasst sich vor allem mit verteidigungs- und rüstungspolitischen Fragestellungen. Das von der Europäischen Union finanzierte Institute for Security Studies (EUISS), die Nachfolgeeinrichtung des früheren WEU-Instituts, soll beratend tätig werden und zum Entstehen eines europaweiten sicherheitspolitischen Diskurses beitragen. Diese Zielsetzung hat sich auch die jüngste Gründung auf seine Fahne geschrieben, das von der Soros Foundation großzügig unterstützte Projekt eines European Council on Foreign Relations.

Schließlich meldet sich mit der Rüstungsindustrie eine wichtige Interessengruppe lautstärker in Brüssel zu Wort. 2004 fusionierten die in den 1970er Jahren gegründete European Defence Industries Group und die 1950 gegründete AECMA (European Association of Aerospace Industries) zusammen mit der EUROSPACE zur AeroSpace and Defence Industries Association of Europe (ASD). Weil rüstungspolitische Kompetenzen bis zur Gründung der ESVP fast ausschließlich auf der nationalen Ebene verblieben waren, führten die Vorgängerorganisationen ein beschauliches Dasein. Dagegen ist die ASD mit etwa 50 Mitarbeitern ein Schwergewicht unter den Brüssler Lobbyorganisationen.

Auch die Rolle der supranationalen Organe wurde aufgewertet. Sicherlich war die europäische Außenpolitik anfänglich kaum institutionalisiert und unterscheiden sich die institutionellen Spielregeln und Einflussmöglichkeiten der europäischen Institutionen im ersten und zweiten Pfeiler bis heute. Die Rollen des Europäischen Parlaments und Gerichtshofs sind beschränkt. Die Europäische Kommission verfügt im zweiten Pfeiler nicht über ein Initiativmonopol. Ihre Hoffnung, es möge gelingen, die Generaldirektion Außenbeziehungen zum Kompetenzzentrum und Ort der Entscheidungsvorbereitung und Umsetzung der europäischen Außenpolitik auszubauen, zerschlug sich spätestens mit der Vertragsrevision von Amsterdam 1997. Stattdessen spielt die institutionelle Musik der GASP/ESVP im Ratssekretariat. Dennoch ist die rasante Institutionalisierung des zweiten Pfeilers die wichtigste Entwicklung der Europäischen Union in der letzten Dekade. Bereits die GASP nach dem Vertrag von Maastricht 1993 hatte nur noch wenig mit der vertrauten Diplomatenrunde der früheren Europäischen Politischen Zusammenarbeit gemein. Die Reformen von Amsterdam und Helsinki 1999 führten zu einem weiteren Institutionalisierungsschub. Trotz der Niederlage der Kommission in Amsterdam weitete sie ihre außen- und sicherheitspolitischen Aktivitäten insbesondere in den Bereichen der gemischten Kompetenzen beträchtlich aus. Im Rat entstand eine ganze Reihe ressourcenstarker Organe: die Institution des Hohen Repräsentanten einschließlich der Policy Unit und des Lagezentrums (SitCen) mit zusammen etwa 60 Mitarbeitern, ${ }^{31}$ die Abteilung E des Generalsekretariats, der Militärstab (EUMS) mit etwa 180 Offizieren, die zivil-militärische Zelle und als neuestes institutionelles Gewächs die 2007 eingerichtete Zivile Planungs- und Führungseinheit (Civilian Planning and Conduct Capability, CPCC). Mit dem Lissabonner Vertrag von 2007 und der geplanten Einrichtung eines Europäischen Auswärtigen Dienstes wird die Institutionalisierung weiter vorangetrieben. Letztere ist für die Herausbildung einer Governance-Struktur in einem doppelten Sinne von Bedeutung. Die internationalen Bürokratien üben selbst dann einen nachhaltigen

31 Christoph Heusgen: Nach den gescheiterten Referenden: Was wird aus dem Außenminister der Union und dem Europäischen Auswärtigen Dienst?, in: integration 4/2005, S. 336-340. 
politischen Einfluss aus, wenn sie sich wie das Generalsekretariat des Rats in erster Linie als Hilfsorgan der Präsidentschaft verstehen. Zudem dienen die Organe der Europäischen Union als Zugangspunkte für NGOs und Interessengruppen zum Entscheidungsprozess.

Soweit sprechen die Entwicklungen gegen die Annahme struktureller Unterschiede zwischen der ersten und zweiten Säule. Einen dritten Einwand, der in der Literatur bisher kaum aufscheint, ${ }^{32}$ halten wir dagegen für offen. Der erste Pfeiler zeichnet sich dadurch aus, dass sich die Betroffenen europäischer Politik innerhalb der Europäischen Union befinden. Sie haben nicht nur ein starkes Motiv, sich zu organisieren und ihre Interessen auf europäischer Ebene geltend zu machen, sondern sie treffen als ,stake holder' auch auf das Entgegenkommen der supranationalen Organe. Insbesondere die Kommission sieht vor dem Hintergrund ihres Demokratieverständnisses in der Zusammenarbeit mit betroffenen Interessengruppen den entscheidenden Baustein für effektives und legitimes Regieren, vorausgesetzt alle von einer Entscheidung betroffenen Interessen haben die Chance, gleichberechtigt an der Formulierung der Entscheidung mitzuwirken. Um ein , level playing field“ der betroffenen Interessen zu schaffen und damit der entscheidenden Voraussetzung für normativ anspruchsvolles Regieren jenseits der Trias von Staatsgebiet, Staatsvolk und Staatsgewalt näher zu kommen, unterstützen und fördern die für den Binnenmarkt zuständigen Generaldirektionen der Kommission mit großem Aufwand zivilgesellschaftliche Organisationen wie NGOs. Sie unterstützen ihre Projekte, tragen zu ihrer Kernfinanzierung bei und haben nicht selten die europäischen, zivilgesellschaftlichen Verbände und Netzwerke als ihre Partner selbst geschaffen. Der erste Pfeiler bildet also nicht zuletzt aufgrund der Empowerment-Strategie der Kommission einen hervorragenden Nährboden für NGOs.

Was die europäische Außen- und Sicherheitspolitik dagegen betrifft, befinden sich die Betroffenen nun einmal außerhalb der Europäischen Union. Während im zweiten Pfeiler die Adressaten europäischer Entscheidungen kaum eine Chance haben, auch zum Autor derselben zu werden, steht die Repräsentativität derjenigen, die sich äußern - in erster Linie NGOs -, aus Sicht der europäischen Organe infrage. Die Kommission interessiert sich zwar für deren Expertise und bedient sich ihrer Dienste bei der Ausführung europäischer Politik - insbesondere im Bereich der Entwicklungshilfe und der humanitären Hilfe greift die Kommission zur Umsetzung ihrer Programme auf NGOs zurück - ist aber äußerst zurückhaltend, was die Institutionalisierung von Konsultationen und die Finanzierung von Kernaktivitäten betrifft.

Wir werden im Folgenden am Beispiel des europäischen Verhaltenskodexes zur Kontrolle von Rüstungsexporten prüfen, ob trotz dieser strukturellen Differenz im Bereich des zweiten Pfeilers ein Zugang von NGOs zum politischen System der Europäischen Union möglich ist, welcher Strategien sich NGOs bedienen und welche Konsequenzen ihr Engagement für die europäischen politischen Prozesse hat.

\section{Der europäische Verhaltenskodex zur Kontrolle von Rüstungsexporten}

Traditionell fällt die Rüstungsexportpolitik ebenso wie die Rüstungsbeschaffung in den Bereich der alleinigen Verantwortung der Mitgliedstaaten. Erst nach dem Ende des OstWest-Konflikts und vor dem Hintergrund der Enthüllungen über das Ausmaß der europäischen Waffenlieferungen an den Irak begannen Diskussionen über eine Europäisierung dieses Politikfeldes durch zwei zusammenhängende Vorhaben: die Liberalisierung des Rüs-

32 Matthias Dembinski: Vom diplomatischen Club zum außenpolitischen Regieren in Netzwerken? Der Einfluss gesellschaftlicher Akteure auf die EU-Außenpolitik, in: Mathias Jopp/Peter Schlotter (Hrsg.): Kollektive AuBenpolitik - Die Europäische Union als internationaler Akteur, Baden-Baden 2007, S. 91-119. 
tungsmarktes und die Harmonisierung der Rüstungsexportrichtlinien. Zu diesem Zweck setzte die Europäische Union 1991 die Arbeitsgruppe für konventionelle Waffen (Working Group on Conventional Arms, COARM) mit dem Mandat ein, Möglichkeiten für ein gemeinsames Vorgehen zu identifizieren. Obwohl sich die Gruppe schnell auf Kriterien für Rüstungsexporte einigte, verhedderten sich die Verhandlungen danach im Dickicht der unterschiedlichen Interessen der großen europäischen Rüstungsexporteure. Dennoch gelang es 1998, den europäischen Verhaltenskodex zur Kontrolle von Rüstungsexporten zu verabschieden. ${ }^{33}$ Er besteht aus acht Kriterien, die die Mitgliedstaaten beachten sollen, wenn sie Rüstungsgüter an Dritte liefern. Darüber hinaus verpflichteten sich die Regierungen in dem Kodex, sich gegenseitig über die Ablehnung von Rüstungsexporten zu informieren. Wenn ein Staat beabsichtigt, ein Rüstungsgeschäft zu tätigen, das bereits von einem anderen abgelehnt worden ist, soll er diesen zuvor konsultieren. Darüber hinaus vereinbarten die Staaten den Austausch von Informationen über ihre Rüstungsexporte im Rahmen der COARM-Arbeitsgruppe, die ihrerseits jährlich einen vertraulichen Bericht für den Rat verfassen sollte. Schließlich sah der Beschluss eine jährliche Bestandsaufnahme vor.

Die Entwicklung des Verhaltenskodexes war von Beginn an von Initiativen des Europäischen Parlaments und einer Gruppe friedenspolitischer NGOs begleitet worden. ${ }^{34}$ Bereits 1991 hatte die britische NGO Saferworld Vorschläge für einen europäischen Kodex entwickelt. ${ }^{35}$ Anfänglich zielte die Kampagne von Saferworld auf das Europäische Parlament, das die Vorschläge der NGOs bei der Formulierung seiner Resolutionen aufnahm. Nachdem die Idee eines Kodexes auf europäischer Ebene verankert war, entwickelte Saferworld in Zusammenarbeit mit zwei weiteren NGOs und einem Team von Völkerrechtlern Vorschläge für präzise Definitionen der Regeln und Prinzipien des Kodexes. ${ }^{36}$ Parallel dazu rief Saferworld die UK Code Working Group ins Leben, die Mechanismen für die wechselseitige Konsultation und den Informationsaustausch zwischen den EU-Staaten entwickelte. Nach dem Wahlerfolg von Labour 1997 knüpfte Saferworld ein Netz mit weiteren NGOs und Think Tanks in anderen europäischen Staaten, das europaweit die nationalen Informationskampagnen koordinierte. ${ }^{37}$

Eine Reihe von Untersuchungen rechnen den NGOs und dem Europäischen Parlament einen Teil des Erfolges zu. ${ }^{38}$ Tatsächlich lässt sich der Einfluss der NGO-Koalition auf Entschließungen des Europäischen Parlaments bis in die Formulierungen hinein nachweisen. Und auch in den Regeln des 1998 beschlossenen Kodexes finden sich an einigen Stellen Vorschläge der NGOs wieder. Umstritten bleibt jedoch, ob das Engagement der NGOs über Formulierungshilfen und die Einflussnahme auf das seinerseits weitgehend machtlose Euro-

33 Rat der Europäischen Union: Verhaltenskodex der Europäischen Union für Waffenausfuhren, Dok. 8675/2/98 REV 2 vom 5. Juni 1998.

34 Jutta Joachim: Von einem intergouvernmentalen zu einem Governance-System? Der Europäische Verhaltenskodex zu Rüstungsexporten und Nicht-Regierungsorganisationen, General International Relations Conference der Deutschen Vereinigung für Politikwissenschaft, Mannheim 2005.

35 Paul Eavis/Owen Greene: Regulating Arms Exports: A Programme for the European Community, London 1991.

36 British American Security Information Council/Saferworld/World Development Movement, in Zusammenarbeit mit Marc Weller/Françoise Hampson: A European Code of Conduct on the Arms Trade, Brussels 1995, wiederabgedruckt in: UNESCO Chair on Peace and Human Rights at Universitat Autónoma de Barcelona (Hrsg.): The Codes of Conduct to Control Arms Transfers, Barcelona 1998, S. 17-21.

37 Ian Davis: The Regulation of Arms and Dual-Use Exports. Germany, Sweden, and the UK, Oxford 2002, S. 100.

38 Siehe beispielsweise Holger Anders: NGOs and the Shaping of the European Union Conventional Arms Regime, in: Elke Krahmann (Hrsg.): New Threats and New Actors in International Security, Basingstoke 2005, S. $177-198$ 
päische Parlament hinaus zu der Wende beitrug, die zur Verabschiedung des Kodexes führte. Um diese Frage zu beantworten, sollen zunächst Erwartungen aus Sicht staatszentrierter Ansätze formuliert werden.

\section{Die Entstehung des europäischen Verhaltenskodexes aus staatszentrierter Perspektive}

Zwei Erwartungen erscheinen plausibel: Erstens Versuche der Regierungen, die internationale Kooperation zu nutzen, um ihre Autonomie zu erweitern und die Mitsprachewünsche der Gesellschaften und Parlamente über Rüstungsexporte abzuwehren. Eine Einschränkung der Transparenz wäre die Folge. Zweitens sollten wir eine Blockade oder ein Ergebnis der Verhandlungen nahe an den Präferenzen des Landes mit den am wenigsten restriktiven Exportkontrollbestimmungen beobachten. Weil dieses Land über einen leichteren Zugang zum Weltmarkt verfügt, eher Skaleneffekte realisieren könnte und weniger restriktive Exportkontrollbestimmungen in einem zunehmend liberalisierten Rüstungsmarkt als Wettbewerbsvorteil wirken, hat es das geringste Interesse an einer Änderung des Status quo.

Für beide Erwartungen gibt es eine Reihe an Indizien: ${ }^{39}$ Beispielsweise nutzte Deutschland die deutsch-französische Rüstungszusammenarbeit oder das multilaterale Rahmenabkommen (Letter-of-Intent) von 1998, um die Entscheidungsspielräume der Exekutive über Rüstungsexporte zu erhöhen. Auch die 1998 festgelegten Regeln des Exportkodexes sahen ursprünglich Vertraulichkeit und eine sehr restriktive Informationspolitik vor. Ebenso finden wir eine Bestätigung der staatszentrierten Annahmen, was die Verhandlungsergebnisse betrifft. Deutschland, dessen Exportkontrollbestimmungen vergleichsweise hohe Hürden setzen, befürwortete eine zeitnahe Regelung auf europäischer Ebene, weil es fürchtete, dass sich die anspruchsvollen deutschen Bestimmungen bei der sich abzeichnenden Liberalisierung der europäischen Rüstungsmärkte als Standortnachteil erweisen würden. Frankreich und Großbritannien, deren Rüstungsindustrien exportabhängiger waren und deren Regelwerke den Transfer von Waffen auch in Spannungsgebiete zuließen, argumentierten dagegen, Rüstungsexporte beträfen den Kern der Staatsraison und müssten folglich ausschließlich von den nationalen Regierungen getroffen und verantwortet werden. In dieser Konstellation konnte sich die Europäische Union 1994 lediglich auf eine Regelung der Exporte von sogenannten dual-use-Gütern verständigen, welche neben ihrer zivilen Anwendung auch zur Herstellung von Massenvernichtungswaffen dienen können. Der Verhaltenskodex zu Rüstungsexporten musste noch vier Jahre warten. Dass er überhaupt verabschiedet wurde, muss aus staatszentrierter Perspektive angesichts dieser Konstellation zunächst überraschen.

Ist dieser Erfolg also den Initiativen des Europäischen Parlaments und der NGOs zu verdanken? Ein genauerer Blick auf die Position der wichtigen Vetospieler Frankreich und Großbritannien nährt Zweifel an dieser Interpretation. In der Tat scheint die Wahl der Labour-Regierung 1997, die vor dem Hintergrund britischer Rüstungsskandale eine Reform der nationalen Exportpolitik hin zu einer ,ethical foreign policy“ angekündigt hatte, für die Verabschiedung des Kodexes wichtiger gewesen zu sein. ${ }^{40}$ Denn um die Verschärfung der Richtlinien innenpolitisch abzusichern, strebte die neue Regierung ihre europäische Einbettung an. ${ }^{41}$ Nicht zufällig orientierten sich die britischen Richtlinien an den acht europäischen

39 Matthias Dembinski/Barbara Schumacher: Wie Europa dem Rüstungsexport Schranken setzt. Von der Zusammenarbeit europäischer Regierungen zum europäischen Regieren, HSFK-Report 9/2005.

40 Duncan Millar: Human Rights: NGOs Urge European Code of Conduct on Arms Sales, IPS-Inter Press Service, 06.03.1997.

41 David Mepham/Paul Eavis: The Missing Link in Labour's Foreign Policy. The Case for Tighter Controls Over UK Arms Exports, London 2002. 
Kriterien und versuchte London umgekehrt, das neue britische Regelwerk zur Grundlage einer europäischen Einigung zu machen.

In der Folge zeigte Paris mehr Flexibilität. Auch in Frankreich schlug die Aufdeckung mehrerer Rüstungsexportskandale hohe Wellen. Zudem erhoffte sich die Regierung von einer europäischen Einbettung der eigenen Rüstungsexportpolitik Rückendeckung gegen amerikanische Strategien, die Politik ihrer Verbündeten auf diesem Feld zu bestimmen. Schließlich ging Paris davon aus, es werde aufgrund der Konsensregel die genaue Ausformulierung des europäischen Regelwerkes entscheiden und zu weit gehende Bestimmungen verhindern können. Tatsächlich enthielt der Ende Mai 1998 von den Außenministern verabschiedete Kodex striktere Regelungen, die bis dahin diskutiert worden waren, nicht mehr. ${ }^{42}$ So ist beispielsweise der Kodex rechtlich nicht bindend, stellt die Frage, ob ein Waffentransfer eines der Kriterien verletzt, in das Ermessen des exportierenden Staates, erlaubt selbst die Durchführung von Geschäften, die ein anderes Land zuvor abgelehnt hat, betont die Vertraulichkeit und verhindert so, dass Regierungen untereinander oder gegenüber ihren Parlamenten und Öffentlichkeiten rechenschaftspflichtig werden. Diese Änderungen wurden auch von NGOs kritisiert, die befürchteten, dass damit das europäische Regelwerk zu einem Instrument der Exekutiven würde, die in einigen europäischen Staaten bestehenden Mitsprachemöglichkeiten der Legislativen und Gesellschaften über nationale Rüstungsexporte einzuschränken. ${ }^{43}$

\section{Entwicklungen nach 1998 - ein Fall für Governance?}

Die Rekonstruktion der politischen Entwicklungen bis 1998 zeigt, dass die Entscheidung über den europäischen Verhaltenskodex zu Rüstungsexporten mit staatszentrierten Modellen vereinbar ist. NGOs waren zwar präsent und konnten sich Gehör verschaffen. Ein direkter Einfluss auf den Beschluss von 1998 ist aber nicht nachweisbar und dieser plausibler mit Veränderungen der Akteurskonstellation zu erklären. Allerdings beobachten wir in der Zeit danach Entwicklungen, die einer staatszentrierten Sichtweise widersprechen und stattdessen die Annahmen von Governance-Ansätzen stützen: a) eine Präzisierung und Verschärfung der Regeln und Normen auf europäischer Ebene, b) eine Zunahme der Transparenz und c) überraschende Anpassungsleistungen auf nationaler Ebene.

Weiterentwicklung der Regeln: Die jährliche Überprüfung des Kodexes eröffnete rüstungskontrollfreundlichen Staaten und NGOs die Möglichkeit, erfolgreich für seine Weiterentwicklung zu werben. Beispielsweise einigten sich die Mitgliedstaaten auf eine gemeinsame Liste der zu kontrollierenden militärischen Güter und definierten zentrale Begriffe des Kodexes wie ,essentially identical transactions“. ${ }^{44}$ Im vierten Jahresbericht sind diese Präzisierungen erstmals in einem ,Kompendium der vereinbarten Verfahrensweisen " niedergelegt. ${ }^{45}$ Darüber hinaus wurden die Regeln des Kodexes auf zusätzliche Tatbestände wie die Vermittlung von Waffengeschäften durch EU-Bürger oder den Transfer von nicht-tangiblen Gütern ausgeweitet.

42 Davis: The Regulation of Arms and Dual-Use Exports, 2002, S. 100.

43 Kyrre Holm: Europeanising Export Controls: The Impact of the European Union Code of Conduct on Arms Exports in Belgium, Germany and Italy, in: European Security 2/2006, S. 213-234.

44 Sibylle Bauer: The EU Code of Conduct on Arms Exports - Much Accomplished, Much to Be Done, 2004, abrufbar unter: http://www.krf.se/download/eu_code_report.pdf (letzter Zugriff: 15.09.2008).

45 Vierter Jahresbericht gemäß Nummer 8 der operativen Bestimmungen des Verhaltenskodex der Europäischen Union für Waffenausfuhren, in: Amtsblatt der Europäischen Union, Nr. C 319 vom 19. Dezember 2002. 
Transparenz: Entgegen der Erwartung aus staatszentrierter Perspektive führte die Europäisierung dieses Politikfeldes zu mehr Transparenz zwischen Regierungen (interne Transparenz) sowie zwischen dem intergouvernementalen Verhandlungsraum und öffentlichen Räumen (externe Transparenz). In Bezug auf die interne Transparenz verbesserten sich sowohl die Quantität als auch die Qualität der Informationen in den jährlichen nationalen Berichten an den Rat nachhaltig. Die Staaten passten das Format ihrer Berichte einander an und gaben untereinander mehr und genauere Informationen über ihre Rüstungsexporte preis. Unter anderem einigten sie sich auf eine schnellere Unterrichtung über abgelehnte Exportanträge und die Einrichtung einer zentralen Datenbasis beim Ratssekretariat. ${ }^{46}$ Schließlich verständigten sie sich 2001 darauf, eine ,trotz einer früheren Verweigerung erteilte Genehmigung" (,undercut") nicht, wie im Kodex vorgesehen, nur dem betroffenen Land, sondern „to the extent compatible with national considerations and on a confidential basis“ allen Mitgliedstaaten mitzuteilen. ${ }^{47}$ Insgesamt notierten selbst kritische NGOs substanzielle Fortschritte. ${ }^{48}$

In Bezug auf die externe Transparenz ist zuallererst die Veröffentlichung der konsolidierten EU-Berichte selbst erwähnenswert, die der Kodex gar nicht vorsieht und die der Rat auf Basis der nationalen Berichte jährlich erstellt. Die Quantität und Qualität der Daten in diesen Berichten nahmen im Laufe der Jahre deutlich zu. War der erste Bericht nur vier Seiten lang und enthielt lediglich unvollständige Globalzahlen zu den erteilten Ausfuhrgenehmigungen und deren Wert sowie den abgelehnten Anträgen und Konsultationen, wurden die Angaben im Laufe der Zeit immer präziser und umfangreicher. Der im Dezember 2005 veröffentlichte Jahresbericht umfasste 288 Seiten und enthielt detaillierte Angaben über die Empfängerländer und exportierten Waffen. ${ }^{49}$ Schließlich folgten mehr und mehr Mitglieder der Europäischen Union dem Beispiel Schwedens und veröffentlichten ihre nationalen Berichte über ihre Rüstungsexportpolitik. ${ }^{50}$

Verschränkung der Ebenen: Drittens beobachten wir erhebliche Anpassungsleistungen auf nationaler Ebene. Selbst Frankreich, ein großer, rüstungsabhängiger und gegenüber dem Kodex skeptisch eingestellter Staat, reformierte und verschärfte als Reaktion auf die europäischen Bestimmungen seine nationale Exportkontrollgesetzgebung. ${ }^{51}$

Für diese Veränderungen ist sicherlich nicht allein und nicht einmal in erster Linie das NGO-Engagement verantwortlich. Ausschlaggebender war vielmehr die Europäisierung dieses Politikfeldes. Damit einher ging zum einen eine Aufwertung institutioneller Rollen wie die der Präsidentschaft. Zum anderen sahen sich die Staaten einer, wenn auch zunächst begrenzten, Rechenschafts- und Begründungserwartung ausgesetzt. Aufgrund dieser Ent-

46 Sibylle Bauer/Mark Bromley: The European Union Code of Conduct on Arms Exports. Improving the Annual Report, SIPRI Policy Paper 8/2004, S. 16.

47 Ebenda; Fünfter Jahresbericht gemäß Nummer 8 der operativen Bestimmungen des Verhaltenskodex der Europäischen Union für Waffenausfuhren, in: Amtsblatt der EU, Nr. C 320 vom 31. Dezember 2003, S. 6.

48 Gemeinsame Konferenz Kirche und Entwicklung (Hrsg.): Rüstungsexportbericht 2004, Bonn/Berlin 2005; Saferworld: Taking Control: The Case for a More Effective European Union Code of Conduct on Arms Exports, 2004, abrufbar unter: http://www.saferworld.org.uk/images/pubdocs/Taking\%20control.pdf (letzter Zugriff: 15.09.2008).

49 Siebter Jahresbericht gemäß Nummer 8 der operativen Bestimmungen des Verhaltskodex der Europäischen Union für Waffenausfuhren, in: Amtsblatt der Europäischen Union, Nr. C 328 vom 23. Dezember 2005, S. 1288.

50 Sybille Bauer: The EU Code of Conduct on Arms Exports - Enhancing the Accountability of Arms Export Policies?, in: European Security 3-4/2003, S. 129-147, hier S. 144.

51 Dembinski/Schumacher: Wie Europa dem Rüstungsexport Schranken setzt, 2005. 
wicklungen ergaben sich neue Handlungsmöglichkeiten für NGOs. Umgekehrt verstärkte ihr Engagement die Effekte der Europäisierung.

So spielte die Institutionalisierung des Kodexes den argumentativen Strategien der NGOs in die Hände. Sie konnten die in der Präambel ausformulierten Zielvorstellungen als europäische Norm darstellen und mit der davon abweichenden Praxis einiger europäischer Regierungen kontrastieren. ${ }^{52}$ Anders als vor 1998 gelang es nun NGOs, die europäische Agenda zu beeinflussen und Staaten rechenschaftspflichtig zu machen.

Mit der Institutionalisierung des Kodexes traten aber auch neue Akteure auf den Plan und mit ihnen potenzielle Koalitionspartner für NGOs. Beispielsweise wurde das Europäische Parlament - ein traditioneller Ansprechpartner der NGOs - aufgewertet, weil sich die finnische Präsidentschaft entschied, den konsolidierten Bericht seinem sicherheits- und verteidigungspolitischen Unterausschuss zuzusenden, der eine Debatte abhielt und Vorschläge zur Weiterentwicklung des Kodexes diskutierte. Ein Jahr später folgte die schwedische Präsidentschaft diesem Beispiel und etablierte damit eine seitdem befolgte Praxis.

Mit der Rechenschafts- und Begründungspflicht wuchs auch der Wert der NGOs als Experten, an die sich sowohl die Präsidentschaften aber auch einzelne Staaten wandten. So arbeitete die britische Labour-Regierung während ihrer Präsidentschaft eng mit einer Gruppe britischer NGOs zusammen ${ }^{53}$ und die holländische Präsidentschaft beauftragte das renommierte Forschungsinstitut Stockholm International Peace Research Institute (SIPRI) mit der Erstellung eines Reports über das nationale Berichtswesen mit dem Ziel, dieses stärker zu harmonisieren. ${ }^{54}$ Auch COARM begann nach 1998 damit, NGOs regelmäßig zu konsultieren.

Schließlich trugen NGOs zu vermehrter Transparenz bei, indem sie die Rüstungsexportpolitiken der Mitgliedstaaten auf europäischer und nationaler Ebene kommentierten und kritisierten sowie Medien, Parlamentarier und Graswurzel-Organisationen in den einzelnen Ländern informierten. Dies lässt sich am Beispiel Frankreichs zeigen, wo sich nach der Institutionalisierung des Kodexes NGOs, die bis dahin ausschließlich im nationalen Rahmen gearbeitet hatten, an den Kampagnen europaweiter Netzwerke beteiligten und somit den französischen öffentlichen Raum gegenüber Europa öffneten. In der Folge wird die restriktive Politik der französischen Regierungen, die Informations- und Mitsprachewünschen der Gesellschaft und des Parlaments traditionell ablehnend gegenüber standen, unmittelbarer mit den liberaleren Praktiken in anderen Ländern vergleichbar und dadurch einem beachtlichen Anpassungsdruck ausgesetzt. Umgekehrt rückt die oft bremsende Politik Frankreichs auf europäischer Ebene deutlicher ins Blickfeld der französischen Gesellschaft und wird so im nationalen Diskurs thematisierbar. In der kommunikativen Verknüpfung beider Ebenen und der daraus resultierenden Sicherung der Rechenschaftspflicht nationaler Regierungen, liegt wahrscheinlich der wichtigste Beitrag der NGOs zum europäischen Regieren.

\section{Schlussfolgerungen}

Die von uns beobachteten Entwicklungen seit der Verabschiedung des europäischen Verhaltenskodexes für Rüstungsexporte sind mit den Erwartungen staatszentrierter Ansätze

52 Amnesty International/BASIC/Christian Aid/Oxfam/Saferworld/World Development Movement: Final Analysis: EU Code of Conduct On The Arms Trade, 1998, abrufbar unter: http://www.basicint.org/WT/armsexp/EUcode-final.htm (letzter Zugriff: 15.09.2008); Saferworld: Taking control, 2004.

53 UK Presidency Project: The British-French Proposal for an EU Code of Conduct on Arms-Exports - briefing paper, 1998; UK Presidency Project: The EU Code of Conduct on Arms-Exports - What Can Your Agency Do?, 1998

54 Gemeinsame Konferenz Kirche und Entwicklung: Rüstungsexportbericht 2004, 2005, S. 34. 
nicht in Übereinstimmung zu bringen, sondern stützen die Annahmen von Governance-Modellen. Dies gilt in besonderer Weise für das Engagement von NGOs. Sie begleiteten, kommentierten und beeinflussten die Entwicklung des europäischen Kodexes. Unterstützt durch institutionelle Entwicklungen gelang es den beteiligten Organisationen, das Informationsmonopol der Exekutiven aufzubrechen, einen öffentlichen europäischen Raum zu schaffen, und diesen mit den nationalen Diskursräumen zu verknüpfen. Weil sich die europäische Politik der nationalen Regierungen nunmehr auf einer öffentlichen Bühne vollzog, konnte sie von einer Fachöffentlichkeit kommentiert und kritisiert werden.

Die Fallstudie weist aber auch auf Unterschiede zwischen der ersten und der zweiten Säule hin. Die Kommission fördert das Heranwachsen einer zivilgesellschaftlichen Basis nur bedingt und versucht nicht durch finanzielle Unterstützung ein ,level playing field“ zu schaffen. Umgekehrt richtet sich das Engagement der NGOs nicht in erster Linie auf die Kommission, sondern stärker auf das Europäische Parlament und die intergouvernementalen Organe wie die Präsidentschaft, die COARM-Arbeitsgruppe oder das Politische und Sicherheitspolitische Komitee. Schließlich ist der normative Anspruch bescheidener, der sich mit der Entwicklung eines Regierungssystems in der GASP verbindet. Es geht nicht um die Hoffnung auf effektives und legitimes Regieren jenseits der Staaten und in Netzwerken betroffener Interessen, sondern um mehr Transparenz, eine Stärkung der Rechenschaftspflicht und eine Verbesserung der Ergebnisse exekutiver Zusammenarbeit durch die Beteiligung von NGOs.

Die Fallstudie über den Verhaltenskodex zeigt, dass NGOs den Kontext und die Ergebnisse der Regierungszusammenarbeit maßgeblich verändern können. Dieser Befund spricht für eine theoretische Öffnung in der GASP. Um die Erklärungskraft von Governance-Ansätzen in der zweiten Säule verlässlicher abschätzen zu können, wären allerdings weitere empirische Arbeiten nötig. Die Menschenrechts-, Entwicklungs- und zivile Krisenpräventionspolitik wäre ein hervorragendes Terrain, um sowohl die Bedeutung als auch die Konsequenzen zivilgesellschaftlichen Engagements zu bestimmen.

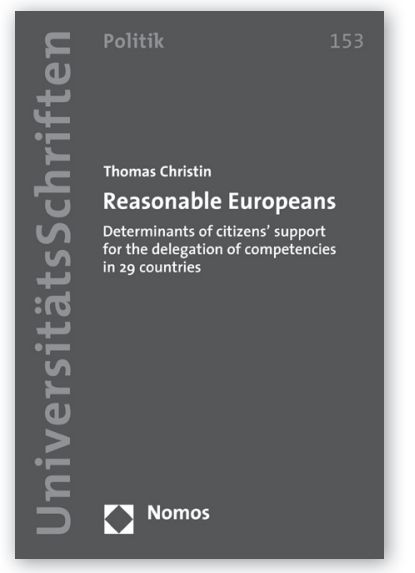

\section{Reasonable Europeans}

Determinants of citizens' support for the delegation of competencies in 29 countries

Von Dr. Thomas Christin

2008, 138 S., brosch., 29,- $€$, ISBN 978-3-8329-3561-O

(Nomos Universitätsschriften - Politik, Bd. 153)

Bitte bestellen Sie bei Ihrer Buchhandlung oder bei Nomos

Telefon 07221/2104-37 | Fax-43 | www.nomos.de | sabine.horn@nomos.de

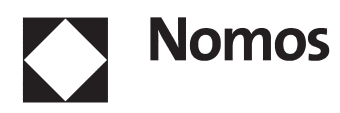

